\title{
Passive compliance for an RC servo-controlled bouncing robot
}

\author{
Friedrich Meyer ${ }^{1}, \quad$ Alexander Spröwitz ${ }^{2} \quad$ and Luc Berthouze ${ }^{1, *}$ \\ ${ }^{1}$ Neuroscience Research Institute, Tsukuba AIST Central 2, Umezono 1-1-1, Tsukuba 305-8568, Japan \\ ${ }^{2}$ Department of Mechatronics, Technische Universität Ilmenau, D-98693 Ilmenau, Germany
}

\begin{abstract}
A novel and low-cost passively-compliant mechanism is described that can be used with RC servos to actuate legged robots in tasks involving high dynamic loads such as bouncing. Compliance is achieved by combining visco-elastic material and metal parts. Joint response to dynamic loads is evaluated using real-world experiments, and force data are obtained from a Lagrangian analysis of the system. The experimental results demonstrate the applicative potential of this mechanism.
\end{abstract}

Keywords: Passive compliance, elastomer spring, robot bouncing, legged robot.

\section{INTRODUCTION}

In humans, most motor behaviours exploit the compliance of each component of the musculo-skeletal system [1]. In the foot, for example, the plantar fascia, together with other ligamentous structures, work to reduce the deformations of the foot's arch under static and dynamic weightbearing. In turn, the foot's arch absorbs dynamic reaction forces during gait [2].

In robots, compliance also has a number of benefits such as lower inertial forces and lower reflected impedance, greater shock tolerance, and potential for efficient (elastic) energy storage and restitution [3]. In large robots with few degrees of freedom (DOFs), compliance has been successfully used in tasks involving high dynamic loads such as hopping, the focus of this study. Raibert's one-leg hopper [4], for example, featured hydraulic actuators and a telescoping leg with an internal air spring. The subsequent Monopod and Uniroo used an articulated leg terminating in a leaf spring instead of an air spring. However, incorporating into an articulated leg system the elastic storage elements vital to good dynamic behaviour posed new locomotion control problems because of asymmetry and high natural frequency. The result of a more integrated approach was CARL, the compliant articulated robot leg [5], which had compliance in each joint of its 3-DOF kinematic chain by way of revolute polymeric springs. The use of an elastomer, combined with electric actuation instead of hydraulic, resulted in a cheaper, smaller and lighter solution with increased stability. Still, controllable passive compliance has yet to show in many of the smaller autonomous robots such as AIBO for example.

The goal of this paper is thus to propose a novel solution that is low-cost, lightweight (about $40 \mathrm{~g}$ ), small enough that it does not prevent its use in articulated systems with many degrees of freedom and 


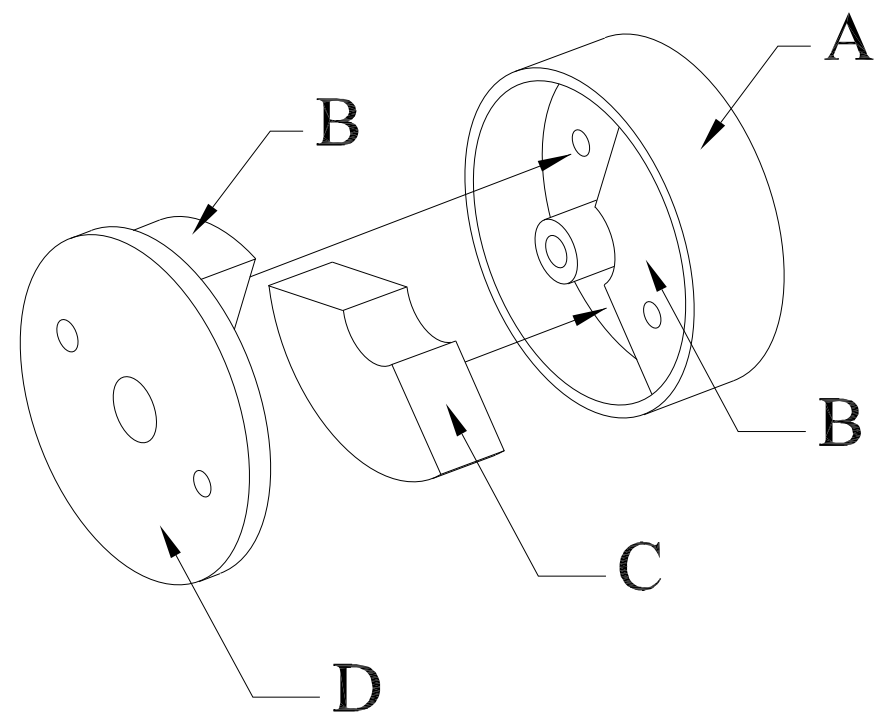

Figure 1: Exploded view of the joint: (A) bush, (B) brass wedge, (C) spring, (D) lid.

does not constrain the mechanical design, and, finally, working in conjunction with as limited actuators as RC servo motors.

\section{JOINT DESIGN}

As shown in Figure 1, the proposed mechanism consists of a brass enclosure (bush and lid) in which an elastomer spring is sandwiched between two brass wedges. One wedge is screwed to the bush, the other to the lid. This arrangement results in an asymmetrically compliant joint that can be mounted in series with an RC servo motor as shown in Figure 2. When the elastomer is compressed, the device is compliant and behaves like a nonlinear torsion spring. Instead, a hard-stop is realised when the two brass wedges press against each other. A fully stiff joint can be obtained by replacing the elastomer spring by a brass wedge.

Since some elastomers (silicone for example) are volume constant, clearance is needed for them to expand. In this study, the brass wedges had a thickness of $6.0 \mathrm{~mm}$ for a bush depth of $6.5 \mathrm{~mm}$. The mold constructed to prepare the elastomer spring was $6.1 \mathrm{~mm}$ deep, meaning that the elastomer was at most $6.0 \mathrm{~mm}$ thick once shrinking due to curing (and subsequently aging) was accounted for. Both brass wedges and elastomer spring had an outer radius of $12.5 \mathrm{~mm}$ and formed an angle of $120^{\circ}$. This shape prevents torsional efforts on the elastomer. Various spring shapes were tested (solid, perforated, or with a neck on the outer rim), however, solid springs were found to yield the best force reaction and therefore this paper only reports results obtained with solid springs.

Although linear springs such as helical or torsional springs could be used as well, this paper will focus on elastomers (silicone rubber and latex) because their nonlinear response to applied force made them more compatible with biological spring systems. The force-compression relationship $(\mathrm{N} / \mathrm{m})$ of 


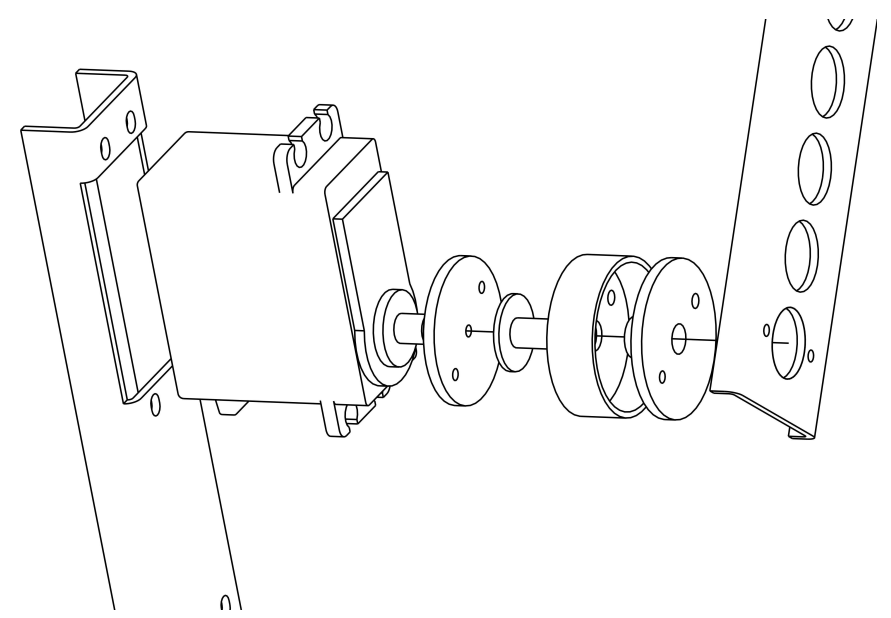

Figure 2: Exploded view of a joint assembly.

the proposed mechanism was estimated by systematically applying forces to the compliant joint and measuring the resulting angular displacement. The response shows an hysteresis and follows a power law on both compression and relaxation phases (Figure 3). Given that the elastomer is volume constant, it is important to note that the exact fitting parameters were not specific to the elastomer itself, but to the whole spring-bush system.

\section{CONTROLLABLE COMPLIANCE}

\subsection{JOINT CHARACTERISTICS}

The compliant system was embedded (Figure 5) in a 3-DOF articulated leg system, and its damping characteristics were qualitatively evaluated during repetitive kicking. The leg was fixed at hip-level to allow for free motion of the lower limbs. The hip motor was programmed to kick back and forth from a set position ( $-135^{\circ}$ with $0^{\circ}$ denoting a forward horizontal position) at a frequency of approximately $0.6 \mathrm{~Hz}$. Other motors were kept stiff in their reset position, and stiff joints were used for both knee and ankle, i.e., the elastomer was replaced by a brass wedge.

The average joint response obtained over 190 kicks shows that the compliant joints are characterized by a large overshoot and high damping, such that there was only one peak after the leg returned to its zero-position (Figure 4L,S). Damping was observed to be higher for silicone springs than for latex springs. In contrast, a stiff joint exhibited several oscillations (Figure 4B), the product of backlash and reflected energy in the joint. In a non-compliant system, this reflected energy typically results in damaging stress to both motors and mechanical structure. 

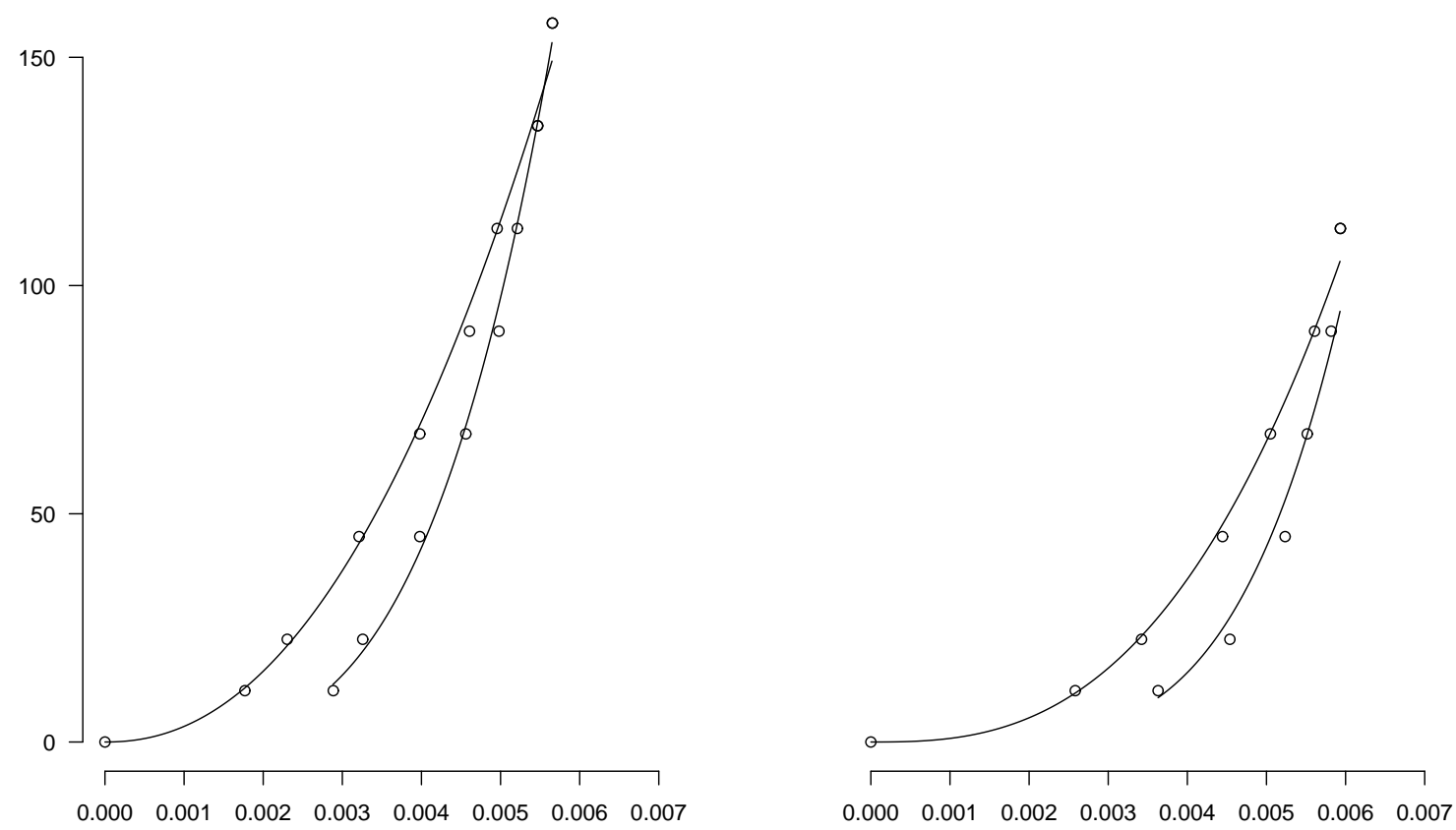

Figure 3: Nonlinear response of the latex-based assembly (left) and silicone-based assembly (right). The data points correspond to quasi-static measurements of the compression (horizontal axis, in $m$ ) in response to a load (vertical axis, in $N$ ), averaged over the 6 springs. The curves show the power law fits.

\subsection{HANDLING OF AN ELASTIC COMPONENT}

The introduction of an elastic component in the system has typically been seen as an obstacle to positional accuracy, stability, and control bandwith $[6,7]$. The ability of the proposed mechanism to handle such component was investigated by adding a springy foot to the articulated leg system. The foot was loosely inspired from human anatomy and featured a rigid heel and two toes made of a flexible bronze alloy. As demonstrated by the bow leg hopping robot [8], such a springy mechanism is very efficient in storing and restituting energy during hopping, with a reported energy recovery rate between hops of $70 \%$.

Compliant bushes and feet were integrated into a lightweight $(1.3 \mathrm{~kg}) 6$-DOF two-legged robot shown in Figure 5 (right). Actuation was provided by 6 high-torque RC servo motors (Hitec HS-945MG). Each joint was assembled so that the force creating element (either the actuated part on ground impact, or the motor torque) would always stress the elastomer, no matter its position in the mechanical chain. The correlate of this design is that any elastic reaction in the other direction (i.e., toward the hard stop) could be neglected, an hypothesis that will be verified later in the Section. Positional motor 


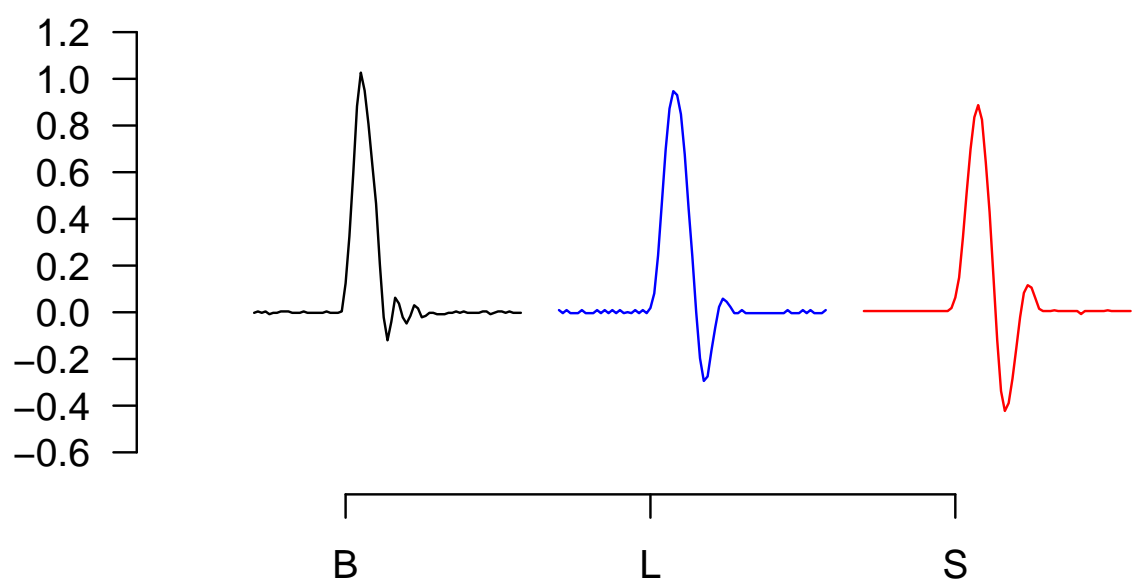

Figure 4: Angular displacement (vertical axis, in radians) of the joint (B: brass, L: latex, S: silicone) in response to a forward kick. The data were realigned to the angle corresponding to the set position. Amplitude differences between the three kicks reflect the compression of the bush in the set position as well as backlash.

commands were generated on a host PC, and sent via serial interface to a microcontroller PIC16F877 for the low-level control of the motors.

Systematic dropping experiments were carried out from a height of $36 \mathrm{~cm}$ as measured from the hip $(23 \mathrm{~cm}$, from the foot). The robot was attached to a parallel guide (Figure 5 , right) to ensure the reproducibility of the falls. Knee and ankle joints were set to $90^{\circ}$ (with small variations due to backlash in the joints) and hip joints were set to $45^{\circ}$ (position shown in Figure 5, right). The trajectories of the joints during the drops were measured by tracking colored markers at a frequency of $30 \mathrm{~Hz}$, and with a spatial resolution of approximately $1 \mathrm{~cm}$.

Figure 6 (top panels) provides a comparison between the behaviour of the system in a stiff configuration (brass wedges in all 6 DOFs) and in a compliant configuration (latex springs on all 6 DOFs). The oscillations induced by the toes (and visible in the stiff configuration) were absorbed within one single bounce in the compliant configuration, which makes it possible to control the robot in the next hop without interference. In addition, an adaptive control algorithm could time the jump phase appropriately to exploit the second part of the impact phase and maximize energy recovery (see [9] for experimental results using Fitzhugh-Nagumo neural oscillators).

To obtain force data, a faithful simulation of the entire system was constructed using Lagrangian 

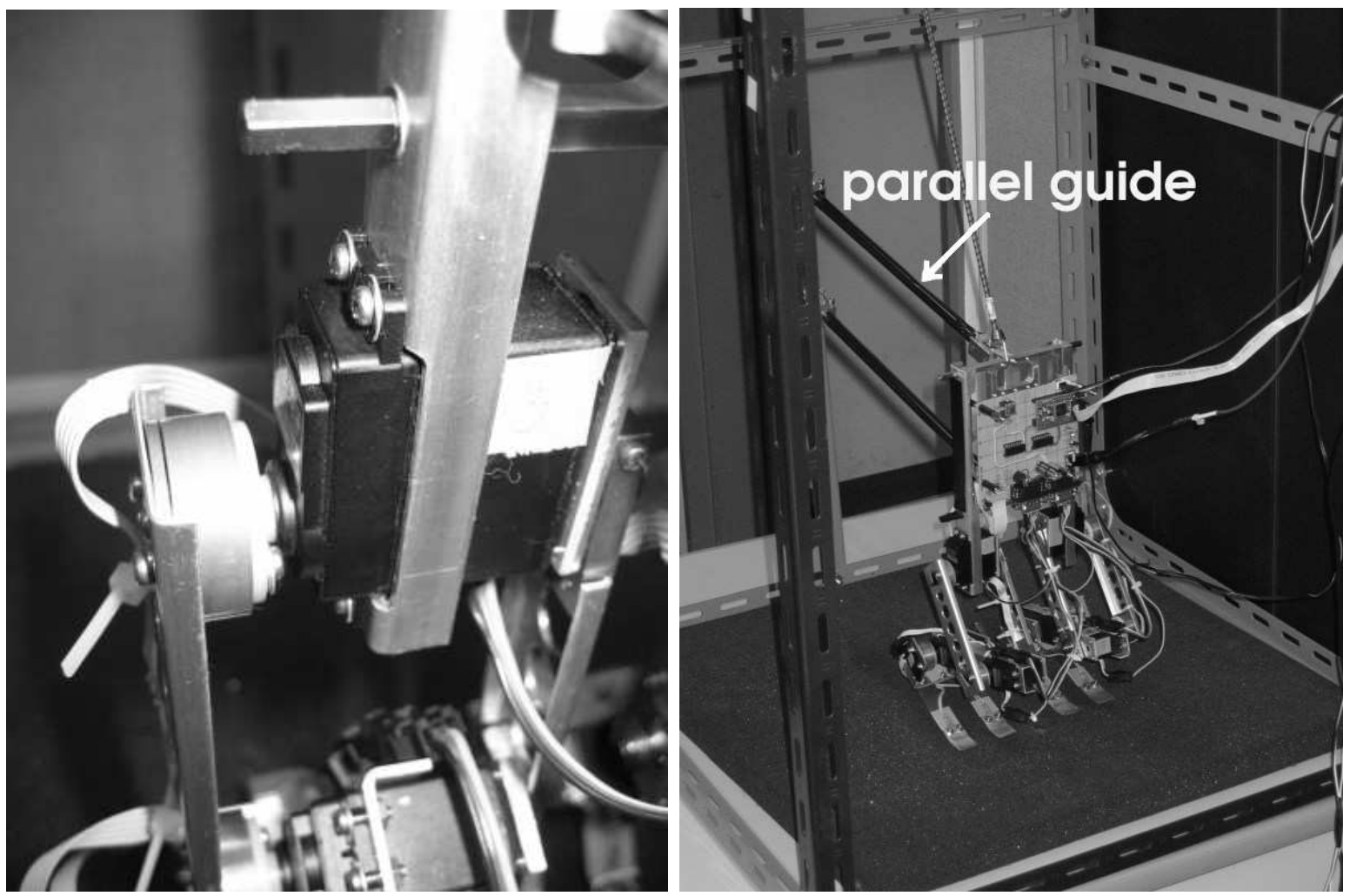

Figure 5: (Left) Joint assembly. (Right) Robot with compliant feet.

analysis. The reader should refer to [10] for a detailed explanation of the simulation setup. Briefly, the damping parameters for both toes and rubber springs were experimentally determined by comparing simulated and actual output. Such approach has been used in the field of biomechanics to implement mathematical models of human mechanics (see [11] for example). Calculations were done with ALASKA (Advanced LAgrangian Solver in Kinetic Analysis, v2.3, Chemnitz Technical University) using RungeKutta integration with a fixed timestep (0.01 milliseconds).

As shown by Figure 6, simulated and actual trajectories were found to match well in the free fall phase, and after impact. Given that the movements, masses and moments of inertia of the simulated parts were identical to those of the real system, it was thus justified to consider the forces in both setups as identical. As expected, high torques were observed in all joints on first impact (Figure 6, bottom panels). In the compliant configuration, no additional peaks were observed. Furthermore, all torques were positive, thus validating the earlier remark that in the context of jumping, it is reasonable to consider impact compensation forces in only one direction.

\section{CONCLUSION}

A simple yet effective damping mechanism was proposed that minimizes the drawbacks typically associated to the use of elastic elements in the kinematic chain. While the flexible foot maximises energy 

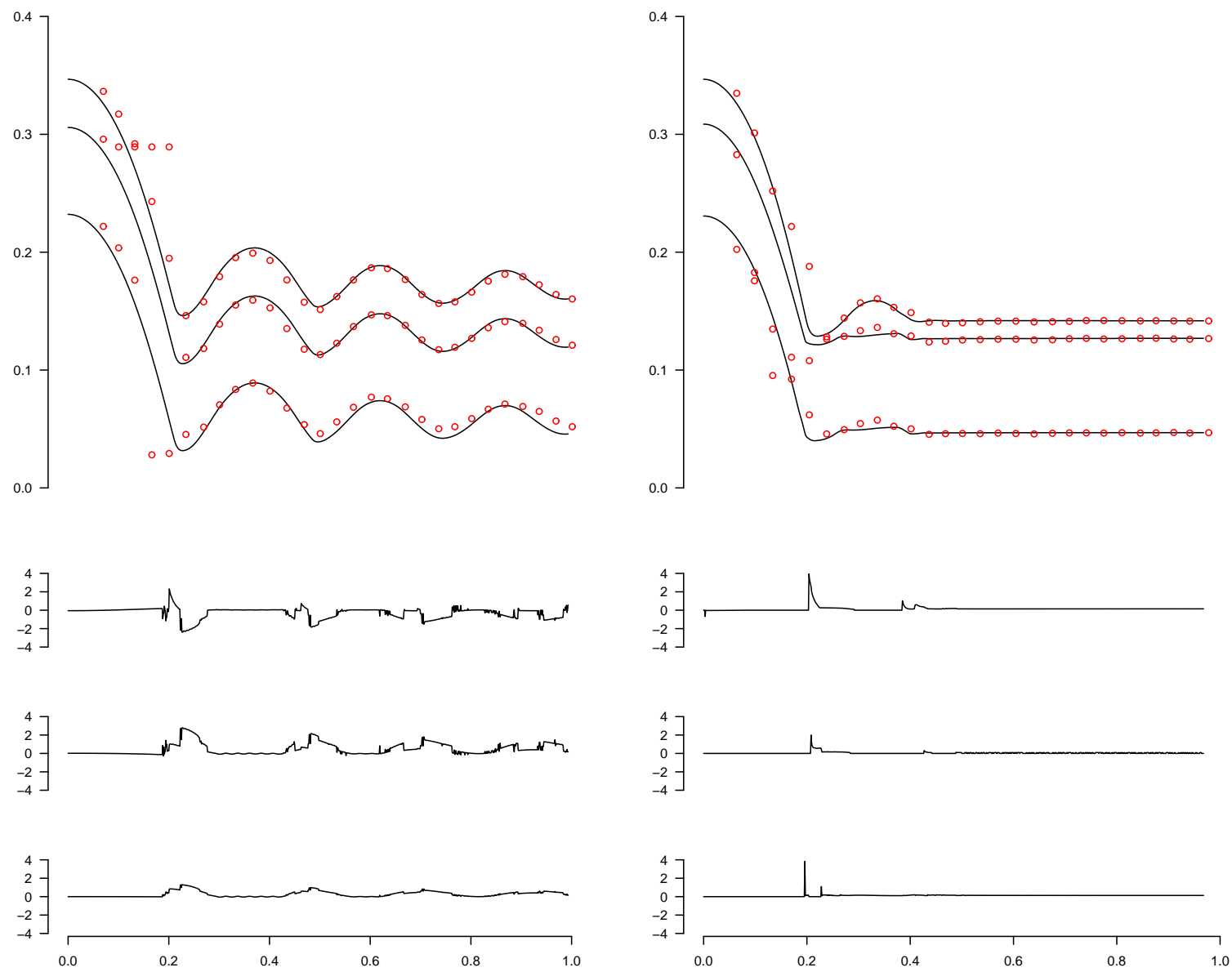

Figure 6: Top panels: Time-series of ankle, knee and hip joint positions (in $m$, vertical axis) during a drop in the stiff (left) and compliant (right) configuration. Latex springs were used in the compliant configuration. In both cases, the simulated data (curves) were time-shifted to overlap with the actual data (points) in the post-impact phase. Bottom panels: Torques (in Nm) in hip, knee and ankle (top to down) determined by simulation. A positive torque is the result of a force that points to the springy part. A negative torque is the result of forces pointing in the direction of the brass-wedge. 
recovery between jumps and reduces the impact-related stress on the mechanical structure and motors, the damping mechanism minimises elastic oscillations and therefore enables accurate control. The mechanism is lightweight and small enough that it can easily be used in small robots, such as RC servo controlled robots. Extensive use of the mechanism should require an investigation of the presence of plastic deformations after extended periods of stress. In addition, a suitable control architecture capable of compensating for the backlash (that translates into delays in the control loop) that affects the joints is necessary. The use of touch sensors under the feet, for example, has been shown to be sufficient to help (asymptotically stable limit-cycle) neural oscillators entrain to the dynamics of the system.

\section{ACKNOWLEDGMENTS}

Friedrich Meyer and Alexander Spröwitz were supported by a MEXT Grant-in-Aid for Young Scientists (no. 15700174) awarded to Luc Berthouze.

\section{REFERENCES}

[1] G. Gottlieb, Muscle compliance: implications for the control of movement, Exercise and Sport Sciences Reviews, 24, 1-34 (1996).

[2] A. Gefen, Stress analysis of the standing foot following surgical plantar fascia release, Journal of Biomechanics, 35(5), 629-637 (2002).

[3] R. Alexander, Elastic Mechanisms in Animal Movement, Cambridge: Cambridge University Press (1988).

[4] M. H. Raibert, Hopping in legged systems - modeling and simulation for the 2d one-legged case, IEEE Transactions on Systems, Man, and Cybernetics, 14, 451-463 (1984).

[5] G. Mennitto and M. Buehler, Carl: A compliant articulated robot leg for dynamic locomotion, Robotics and Autonomous Systems, 18(3), 337-344 (1996).

[6] M. Spong, Modeling and control of elastic joint robots, Journal of Dynamic Systems, Measurement, and Control, 100, 310-319 (1987).

[7] S. Eppinger and W. Seering, Three dynamic problems in robot force control, in Proceedings of IEEE International Conference on Robotics and Automation, pp. 392-397 (1989).

[8] H. Brown and G. Zeglin, The bow leg hopping robot, in Proceedings of the IEEE International Conference on Robotics and Automation, pp. 793-798 (1998).

[9] A. Spröwitz and L. Berthouze, Robust robot bouncing: Passive compliance and flexible phase locking, in Proceedings of 3rd International Symposium on Adaptive Motion in Animals and Machines (to appear). 
[10] F. Meyer, A. Spröwitz, M. Lungarella, and L. Berthouze, Simple and low-cost compliant leg-foot system, in Proceedings of the 2004 IEEE/RSJ International Conference on Intelligent Robots and Systems, pp. 515-520 (2004).

[11] S. Onyshko and D. A. Winter, A mathematical model for the dynamics of human locomotion, Journal of Biomechanics, 13(4), 361-368 (1980). 